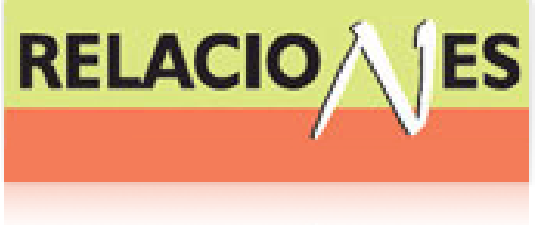

Relaciones. Estudios de historia y sociedad ISSN: 0185-3929

relacion@colmich.edu.mx

El Colegio de Michoacán, A.C

México

Trejo, Zulema; Revilla Celaya, Iván Arturo

El Partido Liberal Sonorense y la candidatura de Ignacio Pesqueira a la gubernatura

Relaciones. Estudios de historia y sociedad, vol. XXXI, núm. 123, 2010, pp. 121-131

El Colegio de Michoacán, A.C

Zamora, México

Disponible en: http://www.redalyc.org/articulo.oa?id=13715893005

- Cómo citar el artículo

Número completo

- Más información del artículo

Página de la revista en redalyc.org

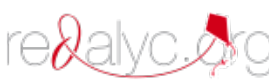

Sistema de Información Científica

Red de Revistas Científicas de América Latina, el Caribe, España y Portugal

Proyecto académico sin fines de lucro, desarrollado bajo la iniciativa de acceso abierto 


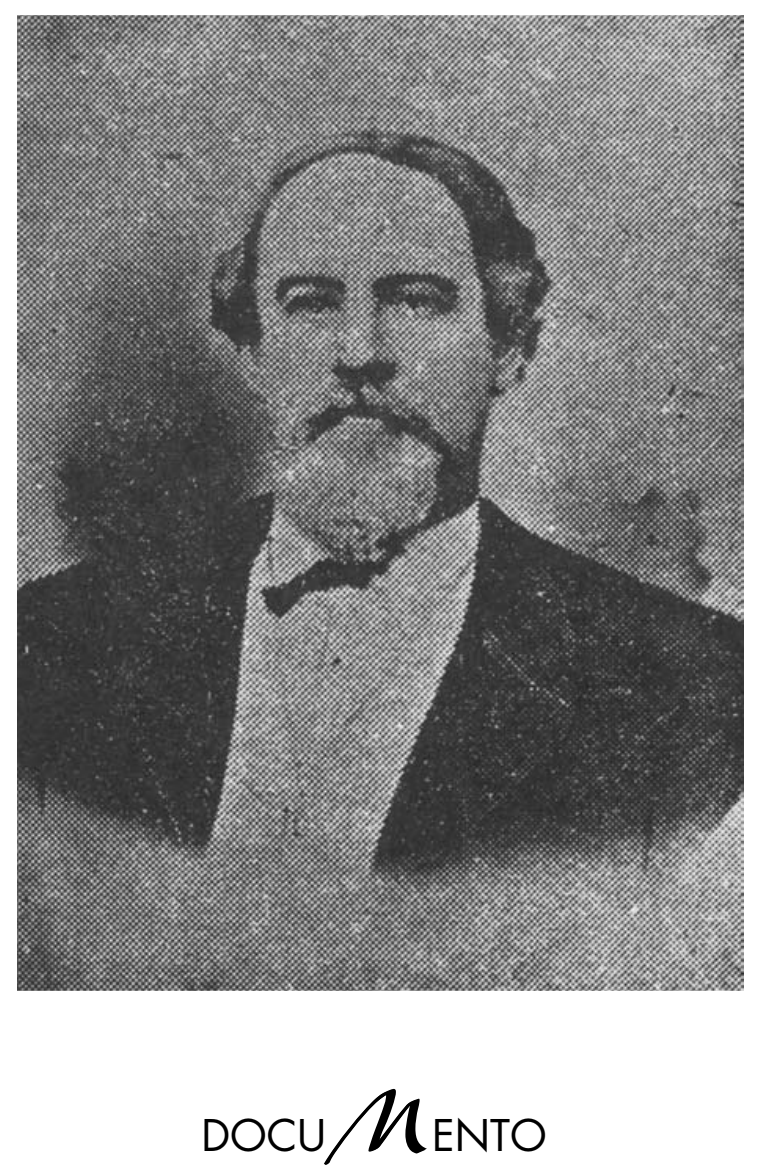

RELACIONES 123, VERANO 2010, VOL. XXXI 


\section{EL PARTIDO LIBERAL SONORENSE \\ Y LA CANDIDATURA DE IGNACIO PESQUEIRA \\ A LA GUBERNATURA}

\section{El Partido Liberal Sonorense}

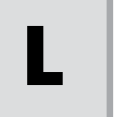

a segunda mitad del siglo XIX es una de las épocas más conflictivas en la historia de nuestro país. Las guerras civiles iniciadas en el ámbito nacional, desde la firma de la independencia y a causa de diferencias ideológicas, tuvieron en cada estado un escenario similar en el cual los principales grupos de poder se disputaron la supremacía política y económica. Estos conflictos, sin embargo, no siempre estuvieron ligados al enfrentamiento entre liberales y conservadores como la historiografía tradicional ha sostenido. El caso de Sonora es representativo al respecto. Aunque los historiadores del siglo pasado identificaron a los partidos liberal y conservador en la entidad, éstos fueron designados sólo de palabra, pues nunca demostraron su existencia como partidos o clubes propiamente establecidos. Lo mismo sucedió con los personajes que fueron etiquetados como liberales o conservadores en la entidad.

El partido conservador no ha sido mencionado mucho en la historiografía local, en cambio el término de conservadores ha sido la palabra clave para designar a Manuel María Gándara y sus seguidores, entre quienes se encontraban "hombres pudientes, y también [...] las facciones proclericales y las diversas tribus del estado". ${ }^{1}$ Pese a ello, esta aseveración no es suficiente para estigmatizar a los gandaristas como conservadores. Si bien enarbolaron el plan de Tacubaya hacia 1858-1859,

${ }^{1}$ Rodolfo Acuña, Caudillo sonorense: Ignacio Pesqueira y su tiempo, México, Ediciones ERA, 1981, p. 40. 
hay que recordar que en 1855 Manuel María Gándara llegó al poder apoyado por los promotores del plan de Ayutla. ${ }^{2}$

La situación cambia cuando se revisa la otra cara de la moneda, es decir a los liberales y el partido liberal de Sonora. Para la época a que nos referimos, mediados del siglo XIX, el historiador Eduardo W. Villa identificó a un grupo de liberales en la ciudad de Ures; mismos que se adhirieron al plan de Ayutla y derrocaron al general Pedro Espejo, quien desempeñaba el ejecutivo sonorense bajo la administración santanista. ${ }^{3}$ El mismo autor señaló la fundación del partido liberal sonorense hacia mediados de 1856. Según su obra, dicho partido fue fundado por el entonces coronel Ignacio Pesqueira. ${ }^{4}$

La mayoría de historiadores posteriores a Villa, que escribieron sobre el XIX sonorense, aceptaron la idea del partido liberal difundida por el autor arriba mencionado. En sus distintas investigaciones identificaron como liberales a aquellos personajes que coadyuvaron a Ignacio Pesqueira a mantenerse en el poder en el lapso que va de 1856 a 1875. Los casos más representativos son las obras de Rodolfo Acuña y Juan Antonio Ruibal Corella. El primero reproduce la cita textual de Villa para señalar la fundación del Partido Liberal Sonorense a manos de Ig-

\footnotetext{
${ }^{2}$ El mismo Manuel María Gándara declaró en algún momento de su vida "que ni aborrezco ni amo al sistema federal, ni al centralismo. Que como ciudadano particular y como funcionario público me adheriré á uno ú otro sistema, tan luego como cualquiera de ellos lo vea adoptado por la Nación, y montado sobre él el Gobierno de mi Pátria". Lo anterior es claro reflejo de que Gándara no se identificaba ni con uno ni con otro bando, sino que apoyaba al que más beneficios le dejara en el aspecto personal. De esto deriva que sus incursiones en contra de las administraciones de José de Aguilar e Ignacio Pesqueira (catalogadas como liberales) buscaran más que la defensa del conservadurismo, la manera de hacerse del poder para él y sus allegados. Manifestación que hace al Gefe Supremo de la República el ciudadano Manuel María Gándara, en la que espresa las causas que le obligaron a salir del Estado de Sonora y las de la revolución de dicho Estado, México, Biblioteca de México, Imprenta de José A. Godoy, 1857, 25.

${ }^{3}$ Eduardo W. Villa, Historia del Estado de Sonora, Hermosillo, Gobierno del Estado de Sonora, 1984, 239.

${ }^{4}$ Sobre su fundación señala "... naciendo en Sonora el Partido Liberal representado por su joven caudillo (Ignacio Pesqueira), que adornado por las virtudes del valor, el patriotismo y la abnegación, lo hacían digno adversario del Comandante Gándara a quien le disputaría palmo a palmo la supremacía en el manejo de la cosa pública, llevando como divisa un noble ideal en vez de ambiciones bastardas". Ibid. p. 244.
} 
nacio Pesqueira. Ruibal Corella, por su parte, va más allá y señala como prominentes liberales a Miguel Urrea, la familia Almada, Crispín S. Palomares, Jesús García Morales y otros personajes que brindaron apoyo económico y material a la administración pesqueirista. ${ }^{5}$

De lo anterior podemos inferir que los historiadores señalaban como liberales a los defensores del gobierno, y como conservadores a aquellas personas que le presentaran oposición. Esto resulta comprensible, en el caso de los liberales, si observamos que la administración de Pesqueira implementó una serie de medidas encaminadas a fortalecer la economía estatal, acabar con la propiedad comunal de los indígenas, abrir el comercio exterior, impulsar la colonización de baldíos y defender la constitución de 1857. No obstante ello, como lo señala la historiadora Zulema Trejo, dichos autores nunca demostraron la existencia de un partido o club liberal Per se. ${ }^{6}$

Los documentos que a continuación presentamos arrojan luz sobre la existencia de un club liberal en la entidad. Este aparece hacia 1861, año en que el general Pesqueira se reeligió por primera vez en el ejecutivo sonorense. ¿Por qué son importantes dichos documentos? Por el hecho de que prueban la existencia de una organización que brindó su apoyo al gobernador, se autodenominó explícitamente liberal y asumió como suyas las ideas liberales que en esa época estaban en boga.

La documentación del club Progresista de Hermosillo y

Respuesta de Ignacio Pesqueira

Los cuatro documentos que transcribimos a continuación son los únicos que hasta el momento se han encontrado en el Archivo General del Es-

\footnotetext{
${ }^{5} J u a n$ Antonio Ruibal Corella, "La época de la reforma" en Historia General de Sonora Tomo III, Hermosillo, Gobierno del Estado de Sonora, 1997, 164-165.

${ }^{6}$ Basándose en autores del siglo XIX dicha historiadora concluyó que en Sonora no existió un partido liberal, aunque ello no significó que tampoco existieron liberales. En tal categoría ubicó a importantes personajes de la vida pública sonorense, tales como José de Aguilar, Fernando Cubillas, Ignacio Pesqueira y Manuel María Gándara. Zulema Trejo Contreras, "Redes, facciones y liberalismo. Sonora, 1850-1876" tesis de doctorado, Zamora, El Colegio de Michoacán, 2004, 94.
} 
tado de Sonora, que prueban la existencia de un grupo de notables que se autodenominaron a sí mismos liberales. El primer documento consta de dos breves comunicados en los cuales se designa a las personas, comisión, que debe presentarse ante el gobernador Ignacio Pesqueira para proponerle que presente su candidatura al gobierno de Sonora. En la documentación lo que más resalta es el hecho de que los integrantes de la comisión, José Monteverde y Antonio Corella, sean personajes que estaban estrechamente vinculados a Pesqueira mediante el parentesco, los negocios y el paisanaje; en otras palabras, se envía como emisarios del club a dos amigos y/o parientes cercanos del gobernador.

El segundo documento es la carta en la cual Ignacio Pesqueira acepta la candidatura que le propone el club Progresista. En ella lo que debe destacarse es la constante alusión del gobernador a la existencia de un partido liberal en Sonora, a las ideas liberales que él y sus aliados profesan, así como a la división del partido liberal, la cual según estudios recientes, equivale a la fragmentación de la facción iñiguista, que dio origen a la facción aguilarista y la pesqueirista. En las elecciones de 1861, a las que se refiere la documentación transcrita, la facción aguilarista presentó como candidato a la gubernatura a Fernando Cubillas.

El tercer documento es un manifiesto que el club progresista posiblemente publicó en el periódico oficial del estado, ${ }^{7}$ o como hoja suelta. En este documento se enumeran las cualidades que hacen a Ignacio Pesqueira la persona ideal para encabezar el gobierno sonorense. Destacan sobre todo sus triunfos en contra de los conservadores en Sinaloa, lo cual en su momento le otorgó prestigio nacional. Asimismo señalan una serie de medidas, todas ellas favorables al grupo de comerciantes que lo apoyaron desde los inicios de su carrera, que lo presentan como un hombre ilustrado y progresista, conocedor de las necesidades de la entidad.

El último documento es, al parecer, una carta que el propio gobernador debía dirigir a personas particulares, creemos que a los notables que

\footnotetext{
${ }^{7}$ Esto no se ha podido corroborar dado que ni la hemeroteca de la Universidad de Sonora ni el Archivo General del Estado de Sonora, conservan ejemplares del Periódico Oficial del Estado para este año.
} 
consideraba podía ganar para su causa, comunicándoles su intención de presentarse como candidato a la gubernatura, el apoyo del club Progresista a su candidatura y los motivos que lo llevaron a optar por reelegirse. Las razones que da para permanecer como titular del ejecutivo en realidad pueden resumirse en una sola, evitar que el poder caiga en manos de la facción aguilarista o la gandarista, porque evitándolo Sonora seguirá fiel a los principios liberales, los cuales, como se ve en todos los documentos, los notables sonorenses integrantes del club progresista y el propio Ignacio Pesqueira, los consideran el camino hacia el progreso y la civilización.

Zulema Trejo

Iván Arturo Revilla Celaya

El Colegio de Sonora

DOCUMENTOS DEL CLUB PROGRESISTA DE HERMOSILLO

PROPONIENDO LA CANDIDATURA DE IGNACIO PESQUEIRA A LA GUBERNATURA $^{8}$

Club Progresista de Hermosillo.-

Resuelto unanimemente, como UU.s [Ustedes] saben, que el C.o [Ciudadano] Ygnacio Pesqueira sea el candidato de este cuerpo para Gobernador del Estado en las proccimas elecciones, ${ }^{9} \mathrm{y}$ resuelto asimismo encomendar á UU.s [Ustedes] la comisión que en nombre y reprsentacion de la misma junta, pase cerca de la del espresado Ciudadano Pesqueira con el fin de suplicarle admita esta candidatura, tenemos la satisfaccion de participarlo á UU.s [Ustedes] para que por su parte admitan tambien y se sirvan cumplir con este acuerdo, con la brevedad que las circunstancias lo ecsijen, admitiendo á la vez las seguridades de nuestra particular

\footnotetext{
${ }^{8}$ Los documentos transcritos a continuación se encuentran en el Archivo General del Estado de Sonora, fondo Ejecutivo, ramo Prefecturas, tomo 369, año 1861.

${ }^{9}$ Se refiere a las elecciones celebradas para elegir gobernador constitucional de Sonora en 1861. Los contendientes fueron el gobernador Ignacio Pesqueira y Fernando Cubillas, quien ocupó el cargo en dos ocasiones en la primera mitad de la década de 1850.
} 
y distinguida amistad.- Dios y Libertad.- Hermosillo, Mayo 22 de 1861.Feliciano Arvizu.- Julian Escalante Srio.- Juan P. Robles Srio.- CC. Lic. José Monteverde y Antonio Corella.- Presentes.

Es copia. Hermosillo, Junio $1^{\circ}$ de 1861.

Julian Escalante, Juan P. Robles

Srios. [Secretarios]

Comisión del Club Progresista de Hermosillo

Ymprenta

Los infrascritos tienen la honra de acompañar á V. E. [Vuestra Excelencia] la credencial que los acredita en comisión para manifestarle que por unanimidad absoluta del Club Progresista V. E. [Vuestra Excelencia] ha sido el candidato para la primera Magistratura del Estado en el p.o [por] bienio constitucional.

La asociacion política á que nos es grato pertenecer, espera que S.E. [Su Excelencia] dando una prueba mas de su constante abnegación y acendrado patriotismo aceptará el alto puesto á que lo llama la voluntad general de los pueblos del Estado. La opinión está bien pronunciada y hace confiar en que V. E. [Vuestra Excelencia] continuará prestando sus relevantes servicios en el ejercicio del poder ejecutivo consolidando p.a [para] siempre la paz de Sonora p.r [por] cuya prosperidad se ha sabido sacrificar mas de una vez, dándole días de gloria y afianzando su respetabilidad asia (sic) fuera de la misma República.

Los q. [que] suscriben p.r [por] sí y á nombre de sus comitentes exigen de V. E. [Vuestra Excelencia] este nuevo sacrificio en pro de nuestro país natal, y á efecto de consolidar su pasificasion y los principios democraticos á cuya sombra prospera; ocupando desde luego el primer lugar entre los listados de la federación.

Dios, Libertad y Progreso. Guaymas, 23 de Mayo de 1861.

José Monteverde

Ant.o Corella [Antonio Corella]

Es copia. Hermosillo $1^{\circ}$ de Junio de 1861

J. P. Robles, Lic Mariano Castro

Srios. [Secretarios]

Esmo. Sor. Gobernador del Estado Ciud.o [Ciudadano] Ygnacio Pesqueira

Presente 


\section{ACEPTACIÓN DE IGNACIO PESQUEIRADE LA PROPUESTA}

DEL CLUB PROGRESITA PARA PRESENTARSE COMO CANDIDTATO

AL GOBIERNODE SONORA

General en Gefe de las Guardias Nacionales en Campaña

Señores

Me ha sido en estremo satisfactorio la ecsitativa que Ustedes se sirven hacerme á nombre del Club Progresista de Hermosillo para que acepte la candidatura de Gobernador constitucional del Estado en las proccimas elecciones.

Aunque he estado resuelto á deponer el mando concluido el periodo constitucional para que fui electo, después de haber prestado á mi país el servicio de pacificar las tribus de indios rebeladas, aunque para mí el poder no tiene mas atractivo que el de poder hacer el bien de Sonora, supuesto que los sinsabores, los desengaños y las defecciones de mu$\operatorname{chos}^{10}$, han sido en gran parte el premio recogido por mis trabajos publicos: sin embargo acepto la candidatura que unánimemente me ofrece el Club de Hermosillo en el que veo representados los principios liberales que hé (sic) defendido dentro y fuera del Estado, mas bien como una prueba de que sé corresponder á una gran confianza, y como una manifestación del deber en que estoy de que el poder no páse (sic) á manos de los enemigos de esos principios ${ }^{11}$, que como un acto que rebeláse ambicion personal de mi parte, ambicion que no poseo, y que debe creérseme que no tiene atractivos para mí cuando el PODER en cinco años que lo he ejercido han sido otros tantos años de sacrificio y penalidades sin otra recompensa que la de haber iniciado la reforma moral y material de nuestro Estado, para que sobre su base se eleve no tarde el edificio de nuestra prosperidad.

\footnotetext{
${ }^{10}$ Es posible que Ignacio Pesqueira se refiera a la fragmentación de la facción iñigista, de la cual surgieron dos agrupaciones: los partidarios de Ignacio Pesqueira y la facción Aguilarista que en las elecciones de 1861 apoyó la candidatura de Fernando Cubillas. Para mayor información al respecto véase Zulema Trejo, "Redes, facciones y liberalismo. Sonora 1850-1876", tesis de doctorado no publicada, El Colegio de Michoacán, 2004, especialmente capítulos 1 y 4 .

${ }^{11}$ Se refiere a los partidarios de Manuel María Gándara agrupados en la facción gandarista, y a los aliados tanto de José de Aguilar como de Fernando Cubillas congregados en la facción aguilarista.
} 
Sírvanse UU.s aceptar las consideraciones de mi aprecio.

Dios y Libertad. Guaymas, Mayo de 1861.

Sres. Lic. D.n José Monteverde y D. Antonio Corella ${ }^{12}$

Comisionados del Club progresista de Hermosillo.

\section{MANIFIESTO DEL CLUB PROGRESISTA EN EL CUAL PROPONE A IGNACIO PESQUEIRA COMO CANDIDATO AGOBERNADOR}

El gran partido liberal del Estado de Sonora propone para Gobernador constitucional en la procsima elección al

\section{C.o [Ciudadano] Ygnacio Pesqueira}

porque fué el primero que en $1856^{13}$ sin elementos de ningún genero se lanzó á combatir la tiranía enraizada en el Estado; por que triunfó de esa tiranía con su valor personal y con la influencia de su prestigio; por que en las subsiguientes revoluciones que han agitado al país, ha combatido como valiente á los que querían sobreponerse con la raza barbara á los hombres que han deseado la libertad de Sonora; por que muchos años atrás y sin mas estimulo que el de hacer un servicio al Estado, hizo personalmente la campaña á los barbaros apaches; por que jamás ha transijido en sus ideas de libertad y de progreso, por que durante su administración combatió á los filibusteros americanos dando á la República el glorioso día de Caborca ${ }^{14}$; por que no vaciló jamás en sostener al Gobierno constitucional de la Nación pronunciandose abiertamente y condenando

\footnotetext{
${ }^{12}$ José Monteverde era hermano de Manuel Monteverde, brazo derecho de Ignacio Pesqueira en la parte administrativa de su gobierno. Salvo estos documentos, no se tenía registrada la participación política activa de José Monteverde. Antonio Corella formó parte de una familia de militares originarios de la zona norte de la entidad, estaba relacionado con Ignacio Pesqueira por vínculos de parentesco, compadrazgo y paisanaje.

${ }^{13}$ En junio de 1856 se produjo el primer levantamiento gandarista de los tres que se produjeron en la segunda mitad de la década de 1850 . Al ser Pesqueira primer vocal del consejo de gobierno, asumió la gubernatura provisional, ya que el gobernador José de Aguilar fue hecho prisionero por los sublevados, y encabezó la defensa del gobierno constitucional hasta la derrota de los gandaristas; posteriormente fue electo gobernador constitucional del estado.

${ }^{14}$ Se refiere a la derrota del filibustero norteamericano Henry Crabb en Caborca en abril de 1857. Pesqueira no encabezó personalmente a la guardia nacional que luchó contra Crabb, pero era el gobernador del estado.
} 
contra el plan de Tacubaya, apesar de los ofrecimientos que le hicieron los reaccionarios, y a pesar también de la oposicion queá su política franca y decidida le hicieron muchos de los que hoy se llaman liberales por que llevó al cabo la toma del puerto de Mazatlán ${ }^{15}$ dando (documento roto) a Sonora, conduciendo personalmente á nuestros valientes nacionales en reñidos encuentros y en acciones dignas de memoria; porque magnanimo y generoso respetó e hizo respetar todos los intereses en el referido puerto, esponiendo su vida; por que salvó la de sus enemigos despues del triunfo: por que de vuelta de la campaña de Sinaloa defendió con valor y dignidad los derechos de la República en las cuestiones graves suscitadas por el capitán Stone y por el comandante Porter de la marina de los Estados Unidos ${ }^{16}$, siendo su conducta elojiada por nacionales y estrangeros; por que ha hecho esfuerzos para reducir á la paz á los barbaros del Norte como la mejor política; porque con la conducta energica en esta ocasión libertó al Estado de ser presa de la ambición de la casa de Jecker y Compañía cuya casa quería apoderarse de todos nuestros terrenos publicos baldios; por que ha hecho esfuerzos para reducir á la paz á los barbaros del Norte como la mejor política que en este respecto puede adoptarse; por que ha tomado medidas capitales para la colonización del Yaqui y $\mathrm{Mayo}^{17}$; por que ha establecido reformas importantisimas en la administracion publica como se vé por la colección de órdenes circulares espedidas en su tiempo; por que ha decretado el derecho de transito á los Estados Unidos; por que ha abierto nuevos puertos al comercio: por que á este ha quitado trabas onerosas cayendo abajo las aduanas interiores y las alcabalas; por que desde el principio de su admón. dejó abolido para siempre en la adunan de Guaymas el monopolio

\footnotetext{
${ }^{15}$ Cuando los conservadores sinaloenses derrocaron al gobernador liberal Plácido Vega, Ignacio Pesqueira se trasladó a Sinaloa al mando de la guardia nacional sonorense para apoyar a los liberales de Sinaloa. La derrota más grande que infringió a los conservadores se produjo en Mazatlán.

${ }^{16}$ Estos personajes se presentaron en Sonora a fines de la década de 1850 para exigir que el gobierno estatal permitiera la medición y deslinde de los terrenos baldíos de la entidad, basados en una concesión otorgada por el gobierno federal a la casa Jecker, Torre y Compañía. Para mayor información véase Rodolfo Acuña, Caudillo Sonorense: Ignacio Pesqueira y su tiempo, México, Era, 1981.

${ }^{17}$ Durante el gobierno de Ignacio Pesqueira se establecieron dos colonias, una en el valle del Yaqui y otra en el del Mayo, ambas fracasaron.
} 
mercantil, el coecho á los empleados que era fuente perenne de desmoralizacion; por que ha establecido mejoras materiales en el Estado como la casa de moneda, el alumbrado publico de las ciudades, la amortizacion y el cambio de la moneda de cobre $\& \&{ }^{18}$; por que debido á sus esfuerzos y á los que ha hecho bajo su dirección el pueblo Sonorense, las ideas de libertad y dignidad han avanzado estraordinariamente, pudiendo asegurarse, que Sonora aunque trabajado por las revoluciones, ocupa un lugar respetable ante los Estados de la federación; por que debido á su patriotismo y rara actividad en tres meses de penosa campaña dejando la primera magistratura ha reducido á los indios Yaquis y Mayos, evolucionandonos la paz que disfrutamos, después de un año de desgracias sin numero, por cuyo ultimo servicio debemos todos los Sonorenses estarle eternamente reconocidos; en fin por que el C.o [Ciudadano] que proponemos lo creemos digno por su conducta, por su carácter y por sus grandes servicios de ocupar el puesto de primer magistrado del Estado, haciendo honor esta elección á Sonora, conosido como es nuestro candidato honrosam.te [honrosamente] dentro y fuera del estado, y dela (sic) República.

\section{CARTA DE IGNACIO PESQUEIRA PARA SOLICITAR APOYO}

\section{A SU CANDIDATURA}

S.D. Y. X.19

Muy señor mío

Por las adjuntas copias se impondrá U. [Usted] de que me he visto obligado á aceptar la candidatura para el Gobierno del Estado en las procsimas elecciones. La razon que he tenido para dar este paso, es la de que mi resolucion tomada anteriormente de separarme definitivamente del poder, fenecido el tiempo que me falta, ha dibidido y debilitado al partido liberal ${ }^{20}$, haciendo posible entonces la elevacion al poder y el

\footnotetext{
${ }^{18}$ Todas estas medidas estuvieron destinadas a favorecer a los comerciantes con los que Pesqueira se alió para derrotar a Manuel María Gándara y sus partidarios. Las alcabalas sí se abolieron por un tiempo, pero al no conseguirse el establecimiento de la contribución directa personal, que debía recaudar lo obtenido por las alcabalas, éstas volvieron a restablecerse.

19 Suponemos que las letras " $Y, X^{\prime \prime}$ representan el nombre de los ciudadanos a los que se les enviaría la carta.

${ }^{20}$ Se refiere al surgimiento de la facción aguilarista que mencionamos anteriormente.
} 
triunfo del partido enemigo vencido en la República y en el Estado. ${ }^{21}$

Nuestros amigos políticos, nuestros compañeros de trabajo, los que infatigablemente han esperado al triunfo de las ideas liberales, unanimemente han conocido el peligro de la división y para salvarlo han ocurrido a mí para que acepte la candidatura de Gobernador, porque han pensado que habiendo tenido yo la satisfaccion de ser el primero que en Sonora se lanzó á poner un dique á la tiranía, y aceptó sin vacilar y de todo corazón, el deber de combatir la reacción dentro y fuera del Estado á costa de sacrificios, han creido repito que estas consideraciones eran bastantes poderosas para colocarme como una bandera que aleja el peligro de que la admón. [Administración] futura del Estado sea entregada en manos de personas que aunque hoy se disfrasan para lograr sus miras de ambicion personal, con el titulo de liberales, la verdad es que sus tendencias y sus trabajos anteriores ${ }^{22}$ deben hacernos comprender que los actuales sean encaminados á otra cosa que á lograr el fin de defender intereses individuales, no bastando como les podía bastar invocar los principios que nosotros hemos defendido á costa de la vida para, ser quéridos (sic) por el pueblo cuyo buen sentido no conseguirán estrabiar.

Las razones espuestas que he creido de mi deber manifestar á U. [Usted] lo persuadirán como lo espero de que solamente el fraccionamiento del partido republicano y liberal en el Estado temido por nuestros amigos, y que podrán comprometer la situación dando un triunfo facil á nuestros enemigos, el temor de que ese triunfo comprometiera la suerte actual y futura del Estado, han sido las que me han obligado solamente á aceptar la candidatura al Gobierno, asegurando á U. [Usted] de la manera mas franca y sincera que ninguna ambicion personal, ninguna mira privada me mueve á cambiar del propósito que he tenido de dejar el poder, sino el deber de contribuir al definitivo establecimiento de la reforma social de nuestro país y al imperio del orden y la paz.

Sírvase U. [Usted] aceptar mi particular atencion (ilegible) Q. B.S.M. [Que Besa Su Mano]

\footnotetext{
${ }^{21}$ Alusión a la facción gandarista, a quien implícitamente identifica como aliada del partido conservador.

${ }^{22}$ Los integrantes de la facción aguilarista estaban vinculados por parentesco y en algunos casos por negocios, con los gandaristas.
} 